\title{
Koide Lepton Relation Explained by Lepton Radius (Compton Wavelength)/4
}

\author{
T. R. Mongan \\ 84 Marin Avenue, Sausalito, CA, USA \\ Email: tmongan@gmail.com
}

How to cite this paper: Mongan, T.R. (2021) Koide Lepton Relation Explained by Lepton Radius (Compton Wavelength)/4. Journal of Modern Physics, 12, 1954-1959. https://doi.org/10.4236/jmp.2021.1214111

Received: November 12, 2021

Accepted: December 14, 2021

Published: December 17, 2021

Copyright $\odot 2021$ by author(s) and Scientific Research Publishing Inc. This work is licensed under the Creative Commons Attribution International License (CC BY 4.0).

http://creativecommons.org/licenses/by/4.0/

\section{Abstract}

The intriguing Koide relation between lepton masses,

$Q_{1}=\frac{m_{e}+m_{\mu}+m_{\tau}}{\left(\sqrt{m_{e}}+\sqrt{m_{\mu}}+\sqrt{m_{\tau}}\right)^{2}} \approx 2 / 3$, involving electron mass $m_{e}$, muon mass $m_{\mu}$, and tau mass $m_{\tau}$, was first presented in 1982 . The reason why $Q_{1} \approx 2 / 3$ has long been unclear. However, describing Standard Model fermions as spheres with radius (Compton wavelength)/4, allows only three fermions in each charge state and avoids the infinite energy density of point particles. Then the Koide parameter $Q_{1} \approx 2 / 3$ because the electron Compton wavelength is more than 200 times the muon and tau Compton wavelengths. A tau mass equivalent of $1776.98 \mathrm{MeV}$, within experimental error bars, would result in $Q_{1}=2 / 3$. The analysis is discussed in the context of efforts to extend the Standard Model of particle physics.

\section{Keywords}

Koide Relation, Standard Model Fermions, Extending the Standard Model

\section{Introduction}

People have wondered about the nature of fundamental particles for centuries. Our best model of fundamental particles at present is the Standard Model of particle physics that identifies the fundamental particles in our universe as twelve spin $1 / 2$ point particles. Those twelve fundamental fermions, all with spin angular momentum $\hbar / 2$ where $\hbar$ is the reduced Planck's constant, are comprised of three charge e leptons, three charge $\frac{2 e}{3}$ quarks, three charge $-\frac{e}{3}$ quarks, where $e$ is the electron charge, and three massless neutrinos with zero charge. Unfortunately, we know the Standard Model is incomplete, because ob- 
servations [1] of neutrino oscillations show that neutrinos must have non-zero mass.

The quantum mechanical uncertainty principle tells us we cannot discern any structure within a particle of mass $m$ that has dimensions smaller than the Compton wavelength $l=\frac{\hbar c}{m c^{2}}$. That does not mean there can be no structure with dimensions less than the Compton wavelength, but only that we can't directly observe it. We can make progress by assuming [2] fundamental fermions in the Standard Model have radius (Compton wavelength)/4, even though we can't directly observe objects of that size. That assumption avoids the problem of the infinite densities of point particles and it has consequences relevant to the Koide relation [3] between lepton masses.

The mass and pressure distribution inside fermions with radius (Compton wavelength) $/ 4$, involving their volume $\sim l^{3}$, their surface area $\sim l^{2}$, and their diameter $l / 2$, results in cubic equations [2] for fermion Compton wavelengths $l$, allowing at most three particles in each charge state. Describing the mass and pressure distribution with surface and linear elements requires minimum surface shell thicknesses and axial core radii on the order of the Planck length $l_{P}=\sqrt{\frac{\hbar G}{c^{3}}}$. The total particle mass is then the sum of the mass equivalents of pressure, $m / 2$, in the volume, the mass equivalent of surface pressure $\frac{\pi}{4} S l^{2}$, and the core mass $L l$. So $\frac{4}{3} \pi \rho\left(\frac{l}{4}\right)^{3}=\frac{4}{3} \pi \frac{\rho}{2}\left(\frac{l}{4}\right)^{3}+4 \pi S\left(\frac{l}{4}\right)^{2}+2 L\left(\frac{l}{2}\right)$. The discriminant $\Delta$ of the resulting cubic equation for fermion Compton wavelengths $A l^{3}-B l^{2}-C l=0$, with $A=\frac{\pi}{96} \rho, B=\frac{\pi S}{4}$, and $C=2 L$, is $\Delta=B^{2} C^{2}-4 A C^{3}$. Since the discriminant $\Delta$ of the cubic equation for fermion wavelengths is positive regardless of the sign of $B$, the cubic equation in $l$ has three real roots corresponding to three fermion Compton wavelengths in each charge state. So, if one were to ask why there are only three lepton masses in the Koide lepton relation, the answer would be that describing Standard Model fermions as objects with radius (Compton wavelength)/4 allows only three leptons.

The background presented above regarding fermions with radius (Compton wavelength)/4 provides some insights into the otherwise somewhat puzzling Koide relation between lepton masses. First, it explains why only three lepton masses can be involved. Second, as shown in the following, the Koide parameter $Q_{1} \approx 2 / 3$ is a consequence of the fact that the electron Compton wavelength is more than 200 times the muon and tau Compton wavelengths.

The following analysis is based on Particle Data Group 2020 data [4] specifying Standard Model fermion masses to six significant figures and the six significant figure value $\hbar c=197.327 \mathrm{MeV} \cdot \mathrm{F}$, where $1 \mathrm{~F}=1 \times 10^{-13} \mathrm{~cm}$, from CODATA 2021 [5] and NIST Reference on Constants, Units, and Uncertainty. This precision is necessary to show why the Koide parameter $Q_{1}$ is so close to 
$2 / 3$.

\section{Koide Q and Lepton Wavelengths}

The lepton mass equivalents ${ }^{4} m_{e}=0.510999 \mathrm{MeV}, m_{\mu}=105.658 \mathrm{MeV}$, and $m_{\tau}=1776.86 \mathrm{MeV}$ define the lepton Compton wavelengths by $l=\hbar c / m c^{2}$. The lepton wavelengths $l_{e}=386 \mathrm{~F}, l_{\mu}=1.87 \mathrm{~F}$, and $l_{\tau}=0.111 \mathrm{~F}$ then determine three parameters $l_{e}, l_{\text {avg }}$, and $\theta_{1}$ related to the three roots of the cubic equation [2] in $l$ that describe the axial, surface, and volume energy distribution within leptons. Then, as seen below, the Koide parameter $Q_{1} \approx 2 / 3$ because the electron Compton wavelength is more than 200 times the muon and tau Compton wavelengths, and a tau mass equivalent of $1776.98 \mathrm{MeV}$, within experimental [4] error bars, would result in $Q_{1}=2 / 3$.

Written in terms of Compton wavelengths, Koide's parameter is

$Q_{1}=\frac{\left(\frac{1}{l_{e}}+\frac{1}{l_{\mu}}+\frac{1}{l_{\tau}}\right)}{\left(\frac{1}{\sqrt{l_{e}}}+\frac{1}{\sqrt{l_{\mu}}}+\frac{1}{\sqrt{l_{\tau}}}\right)^{2}}$. Solutions to the cubic equation [2] in $l$ for leptons with radius $l / 4$, specifying the axial, surface, and volume energy distributions in leptons, are projections on the $l$ axis of vertices of a Nickalls [6] equilateral triangle centered on average lepton wavelength $l_{\text {avg }}=\frac{1}{3}\left(l_{e}+l_{\mu}+l_{\tau}\right)=129.375 \mathrm{~F}$. Those Nickalls wavelengths [6] are $l_{e}=l_{\text {avg }}+R_{1} \cos \theta_{1}$,

$l_{\mu}=l_{\text {avg }}-\frac{1}{2} R_{1} \cos \theta_{1}+\frac{\sqrt{3}}{2} R_{1} \sin \theta_{1}$, and $l_{\tau}=l_{\text {avg }}-\frac{1}{2} R_{1} \cos \theta_{1}-\frac{\sqrt{3}}{2} R_{1} \sin \theta_{1}$, where $\tan \theta_{1}=\frac{l_{\mu}-l_{\tau}}{\sqrt{3}\left(l_{e}-l_{\text {avg }}\right)}, \theta_{1}=0.00394944$, and $R_{1}=\frac{l_{e}-l_{\text {avg }}}{\cos \theta_{1}}=256.773 \mathrm{~F}$, resulting in $Q_{1}=0.666661$. The electron Compton wavelength $l_{e}$ is much greater than the muon Compton wavelength $l_{\mu}$ and the tau Compton wavelength $l_{\tau}$, because the electron mass $m_{e}$ is much less than the muon mass $m_{\mu}$ and the tau mass $m_{\tau}$, resulting in the angle $\theta_{1} \ll 1$. So, $Q_{1} \approx 2 / 3$ results from electron mass less than $1 \%$ of muon or tau mass.

The scale of the equilateral triangle specifying lepton wavelengths is determined by the Nickalls radius $R_{1}$ of the circle circumscribing the triangle centered at $l_{\text {avg }}=129.375 \mathrm{~F}$, and the orientation of the Nickalls triangle is determined by the angle $\theta_{1}$ between the $l$ axis and a line between the center of the circle and the vertex corresponding to the largest Compton wavelength. Koide's parameter $Q_{1}$ is very sensitive to $\theta_{1}$. Fixing $R_{1}$ at $256.773 \mathrm{~F}$ and $l_{\text {avg }}$ at $129.375 \mathrm{~F}$, an increase in $\theta_{1}$ by only $0.007 \%$ to $\theta_{1}=0.003944701$ increases Koide's $Q_{1}$ to $Q_{1}=0.6666666$. That increase in $\theta_{1}$ does not change the electron mass $m_{e}$ or the muon mass $m_{\mu}$, but it does increase the tau mass $m_{\tau}$ slightly to $1776.98 \mathrm{MeV}$, a result within PDG 2020 [4] error bars.

Analogous Koide parameters can be identified for quarks and neutrinos. Quark 
masses $m_{u}=2.16 \mathrm{MeV}, m_{c}=1270 \mathrm{MeV}$, and $m_{t}=173 \mathrm{GeV}$, with charge $2 \mathrm{e} / 3$, and $m_{d}=4.67 \mathrm{MeV}, m_{s}=93 \mathrm{MeV}$, and $m_{b}=4180 \mathrm{MeV}$, with charge $-e / 3$, consistent with PDG 2020 data [4], result in $Q_{2 / 3}=0.85$ and $Q_{-1 / 3}=0.73$. If fundamental fermions are Godel solutions [2] to Einstein's equations and electron neutrino vacuum energy density $=$ (cosmic vacuum energy density), $m_{e v}=0.00136 \mathrm{eV}$. Neutrino oscillation data [1] then estimate $m_{\mu v}=0.00858 \mathrm{eV}$ and $m_{\tau v}=0.0508 \mathrm{eV}$, with neutrino mass sum $0.061 \mathrm{eV}$ about half Vagnozzi's upper bound [7] $0.12 \mathrm{eV}$, and $Q_{0}=0.48$.

\section{Concluding Remarks}

None of the results and conclusions in the preceding discussion, except those related to neutrino mass estimates, are affected by the following remarks. However, we can go further in considering the nature of Standard Model fermions by identifying them with Godel solutions to Einstein's equations rotating with angular velocity $\omega$, with an average matter density $\rho$ equal to the average fermion mass density, pressure $\frac{1}{2} \rho c^{2}$ from negative vacuum energy density $-\frac{1}{2} \rho c^{2}$, and an effective internal gravitational constant $G_{f}=\frac{\omega^{2}}{4 \pi \rho}$. If half of any fundamental fermion charge is located at radius (Compton wavelength)/4 on the axis of rotation, fermion rotation does not cause radiative loss of energy from accelerated charge. The orientation of the fermion rotation axis is unknown until the $\mathrm{z}$ component of fermion angular momentum is measured, so the fermion mass will appear sinusoidally distributed on a disk of radius (1/4) perpendicular to the line of sight.

Three positive Compton wavelengths in each charge state require negative surface mass equivalent density $S$ and positive mass $L$ per unit core length. Negative $S$ results from positive vacuum energy density $\frac{1}{2} \rho c^{2}$ within the shell that is opposite the negative vacuum energy density $-\frac{1}{2} \rho c^{2}$ in the volume. Then the negative pressure equivalent mass inside the shell counters the positive pressure equivalent mass in the volume. So, with no net pressure at the fermion surface, no force acts to increase or decrease fermion size, as necessary for stable fundamental fermions as Godel solutions within our universe. The sum of fundamental fermion mass components is $\frac{\pi}{48} \rho l^{3}$, with surface mass component $-\frac{\pi}{32} \rho l_{\text {avg }} l^{2}$ and core mass component $\frac{\pi}{64} l\left(l_{\text {avg }}^{2}-\frac{R^{2}}{4}\right)$. So, the surface mass multiple of the total mass is $-\frac{3}{2} \frac{l_{\text {avg }}}{l}$ and the core mass multiple of the total mass is $\frac{3}{4} \frac{l_{\text {avg }}^{2}-\frac{R^{2}}{4}}{l^{2}}$. The surface mass and core mass multiples increase in magnitude with decreasing $l$ because smaller fermion volumes at smaller $l$ de- 
crease the volume occupied by positive pressure from negative vacuum energy density of Godel solutions, but the magnitude of the ratio (surface mass)/(core mass) $=-\frac{2 l l_{\text {avg }}}{l_{\text {avg }}^{2}-\frac{R^{2}}{4}}$ increases with $l$.

The internal gravitational constant $G_{l}$ for fundamental fermions with radius $l / 4$, identified as Godel solutions to Einstein's equations, is determined by Godel's relation $\omega=2 \sqrt{\pi G_{l} \rho}$ with the fermion matter density $\rho=\frac{48 \hbar}{\pi c^{2} l^{4}}$ and the angular velocity $\omega$ of the Godel solutions. Fermions with radius $l / 4$ and core radius $r$ have moment of inertia $I=\frac{2}{5} \frac{m}{2}\left(\frac{l}{4}\right)^{2}+\frac{2}{3} \frac{\pi}{4} S l^{2}\left(\frac{l}{4}\right)^{2}+\frac{1}{2} L l r^{2}$. The last term in $I$ is negligible because $r \ll l$, so the resulting internal fundamental fermion gravitational constant is $G_{l}=\frac{3 c^{3} l^{4}}{\hbar\left(l-l_{\text {avg }}\right)^{2}}$.

The angular velocity of the fermion spheres is $\omega=\frac{\hbar}{2 I}=\frac{8 c}{0.2 l-l_{\text {avg }}}$ and the tangential speed $v_{T}$ of points on the spherical shell equator as a multiple of the speed of light is $\frac{v_{T}}{c}=\frac{\omega l}{4 c}=\frac{2 l}{0.2 l-l_{\text {avg }}}$. Closed time-like curves can exist within the Godel solutions corresponding to the lowest mass fermions in each charge state, where $\frac{v_{T}}{C}>1$. Such closed time-like curves would be unacceptable in Godel cosmologies, because they seem to prevent evolution of the cosmos in time. However, they are not problematic in the case of first generation Standard Model fermions that do not evolve in time from their creation to annihilation.

Classical physics idealizes the force of gravity between extended bodies as the force between point masses concentrated at the center of mass of each body. The mathematical construct corresponding to the center of mass of extended Standard Model fermions provides a link to the idealization of point particles in quantum mechanics. At the instant of particle-antiparticle creation, the centers of mass of the respective Godel solutions to Einstein's equations coincide and their spin axes are oppositely aligned. Correspondingly, at the instant of particleantiparticle annihilation, the centers of mass of the respective Godel solutions to Einstein's equations coincide and the spin axes are oppositely aligned.

This description of Standard Model fermions as Godel solutions to Einstein's equations is fully consistent with experimental evidence. Any unfamiliar characteristics of this description of Standard Model fermions should not be a reason for abandoning the model, given the equally unfamiliar (at the time) aspects of quantum mechanical description of atoms, and early indications from general relativity of the existence of black holes.

Future work should focus on efforts to understand the interactions of various Godel solutions to Einstein's equations bearing half of any associated charge at radius (Compton wavelength)/4 on their axis of rotation. Such a simple combi- 
nation of ideas from general relativity and quantum mechanics may provide further insight into the nature of elementary particles, but it is certainly not the long sought unification of general relativity and quantum mechanics. Any such unification must face the fundamental difficulty that general relativity can be seen as a theory of space, while quantum mechanics is a theory describing events within space.

\section{Conflicts of Interest}

The author declares no conflicts of interest regarding the publication of this paper.

\section{References}

[1] Capozzi, F., Lisi, E., Marrone, A., Montanino, D. and Palazzo, A. (2016) Nuclear Physics B, 908, 218. https://doi.org/10.1016/j.nuclphysb.2016.02.016

[2] Mongan, T.R. (2021) Journal of Modern Physics, 12, 983-987. https://doi.org/10.4236/jmp.2021.127060

[3] Koide, Y. (1982) Lettere al Nuovo Cimento, 34, 201-205. https://doi.org/10.1007/BF02817096

[4] Zyla, P.A., et al. (2020) Progress of Theoretical and Experimental Physics, $083 \mathrm{C} 01$.

[5] Tiesinga, E., et al. (2021) Journal of Physical and Chemical Reference Data, 50, 033105. https://doi.org/10.1063/5.0064853

[6] Nickalls, R.W.D. (1993) The Mathematical Gazette, 77, 354. https://doi.org/10.2307/3619777

[7] Vagnozzi, S. (2019) Cosmological Searches for the Neutrino Mass Scale and Mass Ordering. Stockholm University, Stockholm. 